\title{
Mechanical Efficiency at Different Exercise Intensities Among Adolescent Boys With Different Body Fat Levels
}

\section{OPEN ACCESS}

Edited by:

Luca Paolo Ardigò

University of Verona, Italy

Reviewed by:

Pantelis Theodoros Nikolaidis, Hellenic Military Academy, Greece Marko S. Laaksonen,

Mid Sweden University, Sweden

${ }^{*}$ Correspondence:

Georges Jabbour

georgesjabbour1980@hotmail.com

Specialty section:

This article was submitted to

Exercise Physiology

a section of the journal

Frontiers in Physiology

Received: 16 December 2018

Accepted: 28 February 2019

Published: 15 March 2019

Citation:

Jabbour G and Majed L (2019) Mechanical Efficiency at Different

Exercise Intensities Among

Adolescent Boys With Different Body

Fat Levels. Front. Physiol. 10:265.

doi: 10.3389/fphys.2019.00265

\section{Georges Jabbour* and Lina Majed \\ Sport Science Program, College of Arts and Sciences, Qatar University, Doha, Qatar}

This study investigated the mechanical efficiency (ME) and associated factors in obese, overweight, and normal-weight adolescent boys during incremental cycle exercise test to exhaustion. Forty-five sedentary adolescent boys (13-14 years old) were separated in three groups according to the percentage of fat mass as follows: 15 normal-weight (NW) (body fat: $16.0 \pm 1.9 \%$ ), 15 overweight (OW) (body fat: $24.0 \pm 1.6 \%$ ), and 15 obese (OB) (body fat: $31.0 \pm 3.0 \%$ ). All groups completed an incremental cycle exercise to exhaustion in which energy consumption (E, W), ME (\%), lipid oxidation rate (LO, \%), plasma epinephrine and norepinephrine concentrations were determined consecutively at rest and at three intensity levels corresponding to 50 and $75 \%$ of each participant's maximal heart rate (50\%HRmax and 75\%HRmax) and peak oxygen consumption $\left(\dot{V} \mathrm{O}_{2 \text { peak }}\right)$. During the incremental cycle exercise test, plasma epinephrine, and norepinephrine responses as well as ME determined at 50\%HRmax, 75\%HRmax, and at $\mathrm{VO}_{2 \text { peak }}$ stages were significantly lower in $\mathrm{OB}$ compared to $\mathrm{NW}$ and OW individuals ( $p s<0.01)$. Multiple linear regressions showed that body weight $(B=-0.64$, $p<0.001$ ), energy consumption $(B=-0.24, p<0.05$ ) and lipid oxidation $(B=0.69$, $p<0.01$ ) were significant predictors of ME at $50 \%$ HRmax. However, at $75 \% \mathrm{HRmax}$ and

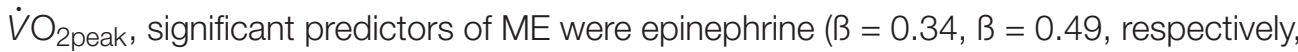
ps $=0.01)$, norepinephrine $(B=0.26, B=0.60$, respectively, $p s<0.05)$ and power output ( $B=0.62, B=0.71$, respectively, ps $<0.01$ ). These findings suggest that excess in body weight exerts a negative effect on $\mathrm{ME}$ at a low intensity by increasing energy consumption for obese and overweight adolescent boys, while at higher intensities (75\%HRmax and $\mathrm{VO}_{2 \text { peak }}$ ) the lower $\mathrm{ME}$ could be better explained by the lower power output and catecholamine responses that were attenuated among obese and overweight adolescent boys.

Keywords: mechanical efficiency, energy consumption, obese, body mass, catecholamine, incremental cycle test to exhaustion, adolescent boys

\section{INTRODUCTION}

Mechanical efficiency (ME) refers to the ability of an individual to transfer the energy consumed into performing external work (Weinstein et al., 2004). ME has recently been investigated as a potential factor underlying metabolic and mechanical adaptations to exercise not only among trained subjects (Boone et al., 2010) but also in special populations (Jabbour et al., 2013, 2017; 
Jabbour and Iancu, 2015). In parallel to other "classical" variables such as cardiovascular risk factors, quality of life, maximal oxygen consumption, ME has been examined as a source of information regarding the effectiveness of exercise interventions (Villelabeitia-Jaureguizar et al., 2018). With this growing interest in using ME for performance and health evaluations there is still a lot to know about underlying key factors.

ME has been proposed as an important measure relating to weight loss and obesity. Indeed, it was suggested that ME is influenced by body weight status (Butte et al., 2007) and metabolic milieu (Jabbour and Iancu, 2015; Laaksonen et al., 2018). A decreased ME may be considered as a limitation for physical activity (Layec et al., 2011; Jabbour and Iancu, 2015), where less efficiency for a given work output is attributed to higher energy consumption and energy cost of breathing during exercise (Layec et al., 2011; Jabbour and Iancu, 2015). For Lafortuna et al. (2006), the decreased ME reported in obese adults may be related to the increased proportion of glycolytic muscle fibers (Kriketos et al., 1997) which are substantially less efficient compared to type I fibers. The latter interpretation was proposed to explain the higher cycling energy cost (Coyle et al., 1992) found in obese adults as compared to normal weight and overweight adults. For Butte et al. (2007), the lower ME values observed for overweight children may be the consequence of the excess in body mass that may limit muscle efficiency. However, the study of Jabbour et al. (2013) conducted on 660 children showed that ME was not affected by body weight status. For these authors, the contradictory lower ME observed in previous studies may be simply related to the method used for ME calculation (net vs. crude value).

More recently, Laaksonen et al. (2018) have investigated the relationship between muscle metabolism and ME among 17 healthy recreationally active male subjects at an intensity corresponding to $45 \%$ of $\mathrm{VO}_{2 \text { peak }}$. Their findings suggested that the use of plasma fatty acids was higher in more efficient subjects and correlated significantly with ME. Non-etheless, no significant differences for blood glucose concentration were observed between the groups suggesting that plasma fatty acids may be an important determinant of ME during submaximal exercises. Furthermore, an interventional study of Jabbour and Iancu (2015) reported an increase in ME following a high intensity training program which was linked to improved homeostasis model assessment estimated insulin resistance and concomitant increases in power output. Interestingly, these improvements were reported at higher intensity stages of an incremental maximal cycling test corresponding to $60 \%, 80 \%$, and $100 \%$ of peak power, respectively. As a factor of performance, ME may be involved in both aerobic and anaerobic performance (Kriketos et al., 1997; Jabbour and Iancu, 2015; Jabbour et al., 2017), therefore indicating that key factors underlying ME may diverge depending on the intensity and task performed.

Most studies on factors contributing to $\mathrm{ME}$ in obese individuals have looked into skeletal muscle adaptations and how they relate to metabolic improvements with training that have been associated for instance with muscle strengthening (Jabbour and Iancu, 2015). However, changes in ME may also be related to hormonal adaptations such as epinephrine and norepinephrine responses. Human studies indicate that obese individuals have reduced catecholamine responses (Jabbour et al., 2011; Vettor et al., 1997) which could significantly affect exercise performance (Strobel et al., 1999). Therefore, the first aim of the present study was to compare ME, metabolic and physiological responses between normal weight, overweight and obese adolescent boys at different intensity levels of an incremental cycle exercise test to exhaustion. The second aim was to examine the relationship between $\mathrm{ME}$ and potential underlying factors amongst which plasma epinephrine and norepinephrine responses. We expect to find the common $\mathrm{ME}$, metabolic and physiological differences as previously reported between the normal weight and obese groups during exercise. As for the relationship between ME and underlying factors, we hypothesize that it would be intensitydependent and that efficiency would be positively correlated to epinephrine and norepinephrine responses during exercise.

\section{MATERIALS AND METHODS}

\section{Participants}

Forty-five healthy adolescent boys were recruited from several high schools in Lebanon. To prevent any maturation variability, only participants in the age range of 13-14 years who were at the same Tanner stage (Stage 3) (Tanner, 1962) were selected. Further inclusion criteria for participation included (i) being sedentary [participating in $<1 \mathrm{~h}$ per week of structured exercise, as assessed by the International Physical Activity Questionnaire (Craig et al., 2003)], (ii) presenting no metabolic, cardiovascular or chronic health problems, (iii) having no history of drug consumption, or (iv) smoking. Health-related information was obtained from the participants' family physician prior to the study. Volunteers were separated into three groups based on the percent body fat (\%body fat) criterion previously described by McCarthy et al. (2006): a normal-weight (NW) group ( $n=15$; \%body fat $<22 \%$ ), an overweight (OW) group $(n=15$; \%body fat $=22-25 \%)$, and an obese (OB) group $(n=15$; \%body fat $>26 \%)$. Participants' physical characteristics and aerobic fitness level are presented in Table 1. Before the start of the experiment, a written informed consent was obtained from the parents and adolescents were familiarized with all testing equipment and procedures. The whole study was approved by the Ethical Committee on Human Research (ECHR) of the University of Balamand (Lebanon) according to the declaration of Helsinki.

\section{Protocol and Materials}

After an overnight fast, participants reported once to the laboratory to perform the protocol that lasted $1 \mathrm{~h}$ on average. They were asked to refrain from strenuous exercise $24 \mathrm{~h}$ before the test. Anthropometric characteristics were firstly measured to assign participants to a weight category group, after which an incremental cycle exercise test followed.

\section{Anthropometric Measurements}

Body mass was measured to the nearest $0.1 \mathrm{~kg}$, with the participants wearing light clothing without shoes, using an electronic scale (MFB 150K100, Kern, Germany). Height was 
TABLE 1 | Physical characteristics and aerobic fitness of participants in the three groups: normal weight (NW), overweight (OW), and obese (OB) adolescents.

\begin{tabular}{|c|c|c|c|c|c|}
\hline & \multirow[t]{2}{*}{$N W(N=15)$} & \multirow[t]{2}{*}{ OW $(N=15)$} & \multirow[t]{2}{*}{ OB $(N=15)$} & \multicolumn{2}{|c|}{ Group effect ( $d f=2)$} \\
\hline & & & & $F$ & $p$ \\
\hline Age (years) & $13.6(0.1)$ & $13.4(0.1)$ & 13.6(0.3) & 1.7 & 0.31 \\
\hline Height (cm) & $162.9(6.2)$ & $164.4(10.4)$ & 168.9(9.6) & 1.6 & 0.33 \\
\hline Body mass (kg) & $50.5(5.2)$ & $67.0(10.0)^{\mathrm{a}}$ & $88.7(14.7)^{a, b}$ & 11.2 & $<0.0001$ \\
\hline $\mathrm{BMI}\left(\mathrm{kg} \cdot \mathrm{m}^{-2}\right)$ & $18.9(1.1)$ & $24.5(1.5)^{\mathrm{a}}$ & $30.8(2.3)^{a, b}$ & 19.1 & $<0.0001$ \\
\hline FM (\%) & $16.0(1.9)$ & $24.5(1.6)^{\mathrm{a}}$ & $31.0(3.0)^{a, b}$ & 23.9 & $<0.0001$ \\
\hline FFM (kg) & $43.0(6.0)$ & $52.0(9.0)^{\mathrm{a}}$ & $62.0(8.0)^{a, b}$ & 21.4 & $<0.0001$ \\
\hline$\dot{\mathrm{V}} \mathrm{O}_{2}$ peak $\left(\mathrm{L} \cdot \mathrm{min}^{-1}\right)$ & $2.10(0.12)$ & $2.36(0.09)^{\mathrm{a}}$ & $2.43(0.11)^{a}$ & 11.8 & $<0.0001$ \\
\hline
\end{tabular}

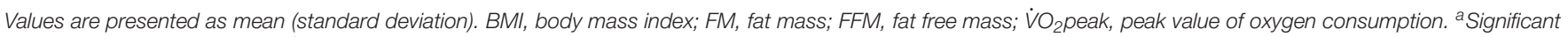
difference with NW $(p<0.01)$. ${ }^{b}$ Significant difference with OW $(p<0.01)$.

determined to the nearest $0.5 \mathrm{~cm}$ with a measuring tape fixed to the wall. The body mass index (BMI, $\mathrm{kg} \cdot \mathrm{m}^{-2}$ ) was calculated as the ratio of body mass $(\mathrm{kg})$ to height squared $\left(\mathrm{m}^{2}\right)$. The \%body fat, referred to here as fat mass (FM, \%) was estimated from 3 skinfold thickness measurement sites (biceps, triceps, and subscapular) according to the validated method of Slaughter et al. (1988) for children and youth. The fat free mass (FFM, kg) was calculated by subtracting the fat mass from the body mass.

\section{Incremental Cycle Exercise Test to Exhaustion}

Participants performed a maximal test on an upright cycle ergometer (Monark Ergomedic 839E, Monark, Sweden) to determine their peak oxygen consumption ( $\dot{V} \mathrm{O}_{2}$ peak). A breathto-breath automated metabolic system (CPX, Medical Graphics, St-Paul, MN, United States) was used to collect gas exchange data. Prior to each test, the system was calibrated according to the manufacturer's instructions using standard gasses of known concentration as well as a calibration syringe for air flow. The laboratory environment was controlled where temperature and relative air humidity were maintained around $23 \mathrm{C}$ and $60 \%$, respectively. Heart rate was continuously measured using a heart rate monitor (Polar-F6, Polar, Finland). At the start of the test, participants remained seated for $5 \mathrm{~min}$ on the bicycle ergometer to measure their resting values. The test started at an initial power of $60 \mathrm{~W}$ and progressively increased by $20 \mathrm{~W}$ every 2 min until exhaustion. During the test, adolescents were instructed to pedal at a rate of 50-70 revolutions per minute that was monitored using an electronic counter (MEV 2000) embedded in the ergocycle. The test was terminated when adolescents could no longer maintain the required pedaling rate $(<40$ revolutions per minute) or requested to stop the exercise. At the end of the protocol, participants were asked to perform an active recovery of $5 \mathrm{~min}$ at $25 \mathrm{~W}$.

At rest and at the end of each intensity level, a venous blood sample was collected from the antecubital vein in a vacutainer tube containing Ethylene Diamine Tetra Acetic Acid (EDTA). Plasma from the venous blood samples was separated by centrifugation at $3000 \times g$ for $20 \mathrm{~min}\left(4^{\circ} \mathrm{C}\right)$ (ORTO ALRESA mod. Digicen.R, Spain). Aliquots were immediately frozen and stored at $-80^{\circ} \mathrm{C}$ for use in subsequent chemical analyses. At the end of incremental test and after a 3-min recovery period, fingertip capillary blood samples were collected and immediately analyzed for blood lactate concentration using a Lactate Pro portable device (Arkray, Japan). This procedure was done to verify one of the test termination criteria.

\section{Data Analysis}

Calculation of Metabolic and Physiological Variables

Gas exchange data were collected on a breath-to-breath basis with a continuous and synchronized measurement of heart rate $\left(\mathrm{HR}\right.$, beats $\left.\cdot \min ^{-1}\right)$. Mean values of $\mathrm{HR}$, oxygen consumption $\left(\dot{V} \mathrm{O}_{2}, \mathrm{~L} \cdot \mathrm{min}^{-1}\right)$, carbon dioxide production $\left(\dot{V} \mathrm{CO}_{2}, \mathrm{~L} \cdot \mathrm{min}^{-1}\right)$, and respiratory exchange ratio (RER) were computed as the average of the last $20 \mathrm{~s}$ of each intensity level where a steadystate was reached. $\dot{V} \mathrm{O}_{2}$ peak was achieved when participants fulfilled at least three of the following criteria: a peak or plateau in $\dot{V} \mathrm{O}_{2}$ values despite an increase in exercise intensity, a RER greater than 1.1, a peak HR above $90 \%$ of the predicted maximal HR (220-age), a blood lactate concentration higher than $8.0 \mathrm{mmol} \cdot \mathrm{L}^{-1}$ and the apparent exhaustion of the subject (Spiro, 1979). In the present work, main variables were assessed at rest and at three stages corresponding to 50 and $75 \%$ of each participant's maximal heart rate $(50 \% \mathrm{HRmax}$ and $75 \%$ HRmax) and at the peak oxygen consumption ( $\dot{V} \mathrm{O}_{2}$ peak) level.

Substrate oxidation was determined at the submaximal aerobic intensity stages (50\%HRmax and 75\%HRmax) based on the corresponding mean values of the non-protein RER. Specifically, the percentage of lipid oxidation (\%LO) contributing to energy was calculated using the method of McGilvery and Goldstein $(1983)$ as follows: $\%$ LO $=[(1-\mathrm{RER}) / 0.29] \times 100$. The percentage of carbohydrate oxidation $(\% \mathrm{CHO})$ was then deduced by subtracting the \%LO from 100 .

\section{Calculation of Mechanical Efficiency}

Net mechanical efficiency $\left(\mathrm{ME}_{\text {net }}, \%\right)$ was calculated using the formula developed by Lafortuna et al. (2006) as the ratio of work performed $(\mathrm{W})$ to the rate of energy consumed (E, W) above resting level, that was in turn computed as follows: $E=(4.94$ $\mathrm{RER}+16.04) \times \dot{V} \mathrm{O}_{2 \text { net }} / 60$ (Garby and Astrup, 1987). Net $\dot{V} \mathrm{O}_{2}$ $\left(\dot{V} \mathrm{O}_{2 \text { net }}, \mathrm{L} \cdot \mathrm{min}^{-1}\right)$ was calculated by subtracting the resting value 
from the gross value at each intensity stage. The resting values of $\mathrm{E}\left(\mathrm{E}_{\text {rest }}\right)$ were also determined based on the equation using

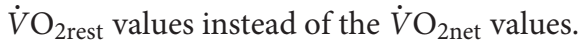

\section{Blood Analyses}

Plasma epinephrine and norepinephrine concentrations were measured using high-performance liquid chromatography (HPLC) (Chromsystems, Thermo finnigan, France), following the method of Koubi et al. (1991). Before the HPLC run, catecholamines were extracted by selective absorption from sodium bisulfite (Chromsystems-HPLC-Kit, Waters, Milford, MA, United States). $1 \mathrm{~mL}$ of plasma previously centrifuged was shaken up briefly with $250 \mu \mathrm{L}$ of sodium bisulfite $(0.25 \%)$ and $50 \mu \mathrm{L}$ internal standard solution $(600 \mathrm{pg}$ dihydroxybenzylamine). After a three times wash (solution TRIS 1M EDTA, pH 8.8), catecholamines were eluted with $120 \mu \mathrm{L}$ buffer $(10 \mathrm{~mL}$ ultra-pure water, $130 \mu \mathrm{L}$ acetic acid, $100 \mu \mathrm{L}$ bisulfate $0.25 \%$ and $25 \mu \mathrm{L}$ EDTA 10\%). Eluant was centrifuged at $4000 \mathrm{rpm}$ for $10 \mathrm{~min}$ (Thermo Fisher Scientific, Jouan. GR412), after which a $50 \mu \mathrm{L}$ of the sample eluant was injected into HPLC column (Column Waters reference 5007 alumina $20 \mathrm{mg}$ ) and eluted with a mobile phase. The flow rate was $1 \mathrm{~mL} \cdot \mathrm{min}^{-1}$ at $13.8 \mathrm{mPa}$ and a potential of $0.60 \mathrm{~V}$. The chromatogram was analyzed by computer integration (Baseline 815, Waters). The detection limit of catecholamines in the described method was $0.06 \mathrm{nM}$ and the inter-assay coefficient of variation was $6.5 \%$. The blood lactate concentration was determined enzymatically using a lactate analyzer (Microzyme, Cetrix, France). Plasma hormones and lactate values were corrected for plasma volume changes using the equation of Van Beaumont (1972).

\section{Statistical Analysis}

Data are presented as mean and standard deviation (SD). After testing for normal distribution (Kolmogorov-Smirnov test), differences between the three groups (i.e., NW, OW, and OB) were analyzed using a one-way analysis of variance (ANOVA) performed on all dependent variables at rest, 50\% HRmax, 75\%HRmax, and $\dot{V} \mathrm{O}_{2 \text { peak. A two-way ANOVA was performed }}$ to further test the interaction effect between the groups and the three relative exercise intensity levels on ME values. Each repeated measures ANOVA was preceded by a Mauchly's sphericity test, and if the test was significant (indicating a violation of the hypothesis of variance homogeneity), a HuynhFeldt correction procedure was used to adjust the degrees of freedom. When needed, a post hoc analysis using NewmanKeul's test was performed for pairwise comparisons. A multiple regression analysis was conducted at each of the studied intensity level (i.e., 50\%HRmax, $75 \% \mathrm{HRmax}$, and $\dot{V} \mathrm{O}_{2 \text { peak }}$ ) to examine the relationship between the $\mathrm{ME}_{\text {net }}$ and various potential predictors (i.e., body weight, power output, energy consumption, lipid oxidation, epinephrine, norepinephrine). The analyses were performed using IBM SPSS Statistics 19 software (IBM SPSS Statistics for Windows, Version 24.0, Armonk, NY, United States: IBM Corp.). A value of $p<0.05$ was accepted as the minimal level of statistical significance.

\section{RESULTS}

\section{Physical Characteristics and Aerobic Fitness Levels}

The age and body height did not differ significantly between the groups of adolescents (Table 1). As expected, results indicated significantly different body mass, BMI and \%FM between each of the three groups $(p s<0.01)$. Furthermore, the FFM $(\mathrm{kg})$ was significantly higher for $\mathrm{OB}$ in comparison to $\mathrm{OW}$ and NW as well as for $\mathrm{OW}$ in comparison to $\mathrm{NW}(p s<0.01)$. The maximal aerobic capacity (absolute $\dot{\mathrm{VO}} \mathrm{O}_{2}$ peak, $\mathrm{L} \cdot \mathrm{min}^{-1}$ ) was significantly higher for the $\mathrm{OB}$ and $\mathrm{OW}$ groups as compared to the NW group $(p s<0.01)$.

\section{Mechanical Efficiency, Metabolic and Physiological Variables Resting Values}

Resting values of oxygen consumption were significantly higher for the OB group in comparison to the NW and OW groups, as well as for the OW as compared to the NW ( $p<0.01$, Table 2). However, no significant differences were revealed between groups for resting values of lactate concentration, heart rate, RER, and epinephrine and norepinephrine concentrations.

\section{Submaximal Exercise Intensities}

At the studied moderate intensity (i.e., 50\%HRmax), most of the variables differed significantly between each of the three groups even though this relative intensity induced similar values of power output (PO), heart rate and lactate concentrations in all groups (Table 3). For instance, higher oxygen and energy consumption, RER and \%LO and lower \%CHO, epinephrine and norepinephrine concentrations were seen in the $\mathrm{OB}$ group as compared to the OW and NW groups ( $p s<0.01)$, as well as for the OW in comparison to the NW group ( $p s<0.01$ ).

At $75 \%$ HRmax, the oxygen and energy consumption values as well as the heart rate, RER and lactate concentrations did not differ statistically between the three groups although power output was significantly higher for the NW group as compared to both OW and OB groups (Table 3). Moreover, the $\mathrm{OB}$ group showed a significantly lower increase in epinephrine and norepinephrine compared to the NW and OW groups $(p s<0.01)$.

\section{Peak Exercise Intensity Values}

At $\dot{V} \mathrm{O}_{2 \text { peak }}$, all studied variables differed significantly between each of the three groups, except for heart rate and RER values (Table 4). Specifically, the NW group reached the highest power output $\dot{V}$ and presented the lowest $\dot{V} \mathrm{O}_{2 \text { peak }}$ and lactate concentration as compared to the OW and $\mathrm{OB}$ groups $(p s<0.01)$. The $\mathrm{OB}$ and $\mathrm{OW}$ groups presented significantly different epinephrine and norepinephrine levels that were lower when compared to the NW groups ( $p s<0.01$ ).

\section{Mechanical Efficiency}

The two-way ANOVA on $\mathrm{ME}_{\text {net }}$ revealed significant main effects of group $[F(2,28)=102.95, p<0.001$, 
TABLE 2 | Metabolic and physiological responses at rest for the three groups: normal weight (NW), overweight (OW), and obese (OB) adolescents.

\begin{tabular}{|c|c|c|c|c|c|}
\hline & \multirow[t]{2}{*}{ NW $(N=15)$} & \multirow[t]{2}{*}{ OW $(N=15)$} & \multirow[t]{2}{*}{ OB $(N=15)$} & \multicolumn{2}{|c|}{ Group effect $(d f=2)$} \\
\hline & & & & $\boldsymbol{F}$ & $p$ \\
\hline$\dot{V} \mathrm{O}_{2 \text { rest }}\left(\mathrm{L} \cdot \mathrm{min}^{-1}\right)$ & $0.19(0.01)$ & $0.35(0.02)^{a}$ & $0.45(0.02)^{a, b}$ & 11.8 & $<0.0001$ \\
\hline Lactate $\left(\mathrm{mmol} \cdot \mathrm{L}^{-1}\right)$ & $1.3(0.3)$ & $1.3(0.5)$ & $1.3(0.7)$ & 1.3 & 1.53 \\
\hline HR (beats.min ${ }^{-1}$ ) & $78.3(2.2)$ & $76.2(9.1)$ & $77.4(3.1)$ & 2.9 & 0.44 \\
\hline RER & $0.78(0.09)$ & $0.79(0.09)$ & $0.78(0.09)$ & 2.2 & 0.22 \\
\hline$E_{\text {rest }}(\mathrm{W})$ & $69(11)$ & $79(17)^{\mathrm{a}}$ & $99(15)^{a, b}$ & 6.6 & $<0.01$ \\
\hline Epinephrines $\left(\mathrm{nmol} \cdot \mathrm{L}^{-1}\right)$ & $0.85(0.01)$ & $0.81(0.02)$ & $0.82(0.01)$ & 1.9 & 0.24 \\
\hline Norepinephrines (nmol. $\mathrm{L}^{-1}$ ) & $2.50(0.01)$ & $2.40(0.04)$ & $2.60(0.03)$ & 3.9 & 0.74 \\
\hline
\end{tabular}

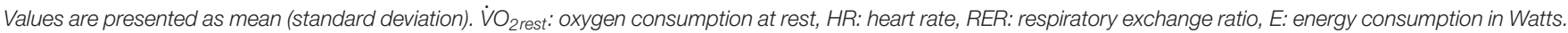
a Significant difference with NW ( $p<0.01)$. b Significant difference with OW $(p<0.01)$.

TABLE 3 | Metabolic and physiological responses at the studied submaximal intensity levels (50\%HRmax, 75\%HRmax) of the incremental cycle exercise test to exhaustion for the three groups: normal weight (NW), overweight $(\mathrm{OW})$, and obese $(\mathrm{OB})$ adolescents.

\begin{tabular}{|c|c|c|c|c|c|}
\hline & \multirow[t]{2}{*}{ NW $(N=15)$} & \multirow[t]{2}{*}{ OW $(N=15)$} & \multirow[t]{2}{*}{ OB $(N=15)$} & \multicolumn{2}{|c|}{ Group effect $(d f=2)$} \\
\hline & & & & $\boldsymbol{F}$ & $p$ \\
\hline \multicolumn{6}{|l|}{ At $50 \%$ HRmax } \\
\hline$\dot{V} \mathrm{O}_{2 \text { net }}\left(\mathrm{L} \cdot \mathrm{min}^{-1}\right)$ & $1.32(0.02)$ & $1.68(0.02)^{\mathrm{a}}$ & $1.81(0.02)^{a, b}$ & 42.1 & $<0.0001$ \\
\hline $\mathrm{PO}(\mathrm{W})$ & $80(5)$ & $80(10)$ & $80(10)$ & 1.3 & 0.31 \\
\hline Lactate $\left(\mathrm{mmol} \cdot \mathrm{L}^{-1}\right)$ & $3.9(1.9)$ & $3.8(1.7)$ & $4.1(1.1)$ & 2.6 & 0.36 \\
\hline HR (beats.min ${ }^{-1}$ ) & $104.7(2.1)$ & $106.2(3.1)$ & 102.9(6.1) & 0.6 & 0.54 \\
\hline RER & $0.78(0.05)$ & $0.82(0.04)^{a}$ & $0.89(0.07)^{a, b}$ & 11.2 & $<0.01$ \\
\hline$\%$ LO & $48(2)$ & $39(1)^{\mathrm{a}}$ & $22(3)^{a, b}$ & 20.2 & $<0.0001$ \\
\hline$\% \mathrm{CHO}$ & $52(2)$ & $61(1)^{\mathrm{a}}$ & $78(3)^{a, b}$ & 20.1 & $<0.0001$ \\
\hline$E(W)$ & $458(11)$ & $562(32)^{a}$ & $615(14)^{a, b}$ & 30.1 & $<0.0001$ \\
\hline Epinephrines $\left(\mathrm{nmol} \cdot \mathrm{L}^{-1}\right.$ ) & $1.61(0.1)$ & $0.98(0.2)^{\mathrm{a}}$ & $0.82(0.1)^{a, b}$ & 8.6 & $<0.01$ \\
\hline Norepinephrines $\left(\mathrm{nmol} \cdot \mathrm{L}^{-}\right.$) & $5.4(0.3)$ & $4.2(0.3)^{\mathrm{a}}$ & $3.9(0.4)^{a, b}$ & 19.4 & $<0.001$ \\
\hline \multicolumn{6}{|l|}{ At $75 \%$ HRmax } \\
\hline$\dot{V} \mathrm{O}_{2 \text { net }}\left(\mathrm{L} \cdot \mathrm{min}^{-1}\right)$ & $2.01(0.04)$ & $2.02(0.02)$ & $2.01(0.03)$ & 2.1 & 0.22 \\
\hline $\mathrm{PO}(\mathrm{W})$ & $140(5)$ & $120(10)^{a}$ & $120(10)^{\mathrm{a}}$ & 11.8 & $<0.001$ \\
\hline Lactate $\left(\mathrm{mmol} \cdot \mathrm{L}^{-1}\right)$ & $6.9(1.9)$ & $6.8(1.7)$ & $6.1(1.1)$ & 2.6 & 0.36 \\
\hline HR (beats.min ${ }^{-1}$ ) & $144.2(2.2)$ & $152.2(2.1)$ & $148.9(2.1)$ & 0.9 & 0.56 \\
\hline RER & $0.89(0.05)$ & $0.87(0.04)$ & $0.87(0.07)$ & 1.6 & 0.64 \\
\hline \%LO & $8(2)$ & $9(1)$ & $8(3)$ & 11.2 & 0.46 \\
\hline$\% \mathrm{CHO}$ & $92(2)$ & $91(1)$ & $92(3)$ & 3.1 & 1.1 \\
\hline$E(W)$ & $685(12)$ & $684(12)$ & $681(19)$ & 2.1 & 2.2 \\
\hline Epinephrines $\left(\mathrm{nmol} \cdot \mathrm{L}^{-1}\right)$ & $2.01(0.1)$ & $1.81(0.2)$ & $1.51(0.4)^{\mathrm{a}, \mathrm{b}}$ & 6.6 & $<0.01$ \\
\hline Norepinephrines $\left(\mathrm{nmol} \cdot \mathrm{L}^{-1}\right.$ ) & $7.4(0.2)$ & $5.9(0.3)^{a}$ & $5.3(0.1)^{a, b}$ & 11.1 & $<0.001$ \\
\hline
\end{tabular}

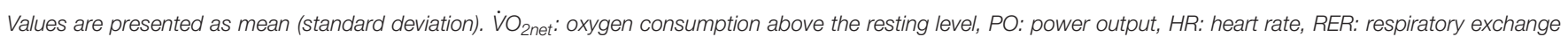

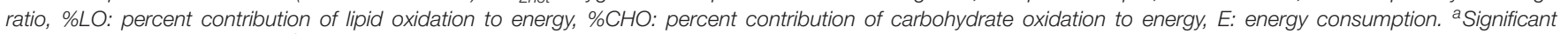
difference with NW $(p<0.01)$. ${ }^{b}$ Significant difference with OW $(p<0.01)$.

$\left.\eta^{2}=0.88\right]$ and intensity $[F(2,28)=137.03, p<0.001$, $\left.\eta^{2}=0.91\right]$. No significant interaction effect was found between factors. Post hoc comparisons showed that $\mathrm{ME}_{n e t}$ differed significant between all groups and all intensities $(p s<0.01)$ with the smallest values found for the $\mathrm{OB}$ group as well as for the lowest studied intensity level (Figure 1).

Multiple linear regressions were calculated to examine the degree to which studied variables predicted $\mathrm{ME}_{\text {net }}$ at each studied intensity level. Significant regression equations were found at $50 \% \mathrm{HRmax}\left[F(2,42)=20.25, p<0.01, R^{2}=0.47\right]$, $75 \%$ HRmax $\left[F(2,42)=4.14, p<0.05, R^{2}=0.19\right]$ and $\dot{V} \mathrm{O}_{2 \text { peak }}\left[F(3,35)=11.01, p<0.01, R^{2}=0.48\right]$. Specifically, at $50 \%$ HRmax, body weight $(B=-0.64, p<0.001)$, energy consumption $(\beta=-0.24, p<0.05)$ and lipid oxidation $(B=0.69, p<0.01)$ were significant predictors of $\mathrm{ME}_{\text {net. At }} 75 \% \mathrm{HRmax}$, the analysis demonstrated that the epinephrine $(B=0.34, p=0.01)$, norepinephrine $(B=0.26, p=0.01)$ and power output $(B=0.62, p<0.01)$ contributed significantly to $\mathrm{ME}_{\text {net }}$. Finally, at $\dot{V} \mathrm{O}_{2 \text { peak }}$, 
TABLE 4 | Metabolic and physiological responses at the peak intensity level ( $\mathrm{VO}_{2}$ peak) of the incremental cycle exercise test to exhaustion for the three groups: normal weight (NW), overweight (OW), and obese (OB) adolescents.

\begin{tabular}{|c|c|c|c|c|c|}
\hline & \multirow[t]{2}{*}{ NW $(N=15)$} & \multirow[t]{2}{*}{ OW $(N=15)$} & \multirow[t]{2}{*}{ OB $(N=15)$} & \multicolumn{2}{|c|}{ Group effect $(d f=2)$} \\
\hline & & & & $\boldsymbol{F}$ & $p$ \\
\hline$\dot{V} \mathrm{O}_{2 \text { peak }}\left(\mathrm{L} \cdot \mathrm{min}^{-1}\right)$ & $2.10(0.12)$ & $2.36(0.09)^{a}$ & $2.43(0.11)^{a}$ & 11.8 & $<0.0001$ \\
\hline $\mathrm{PO}(\mathrm{W})$ & 180(19) & $160(10)^{a}$ & $160(15)^{a}$ & 19.8 & $<0.001$ \\
\hline Lactate $\left(\mathrm{mmol} \cdot \mathrm{L}^{-1}\right)$ & $8.4(4.4)$ & $8.9(3.4)$ & $9.8(3.3)^{a, b}$ & 21.32 & $<0.001$ \\
\hline HR (beats·min ${ }^{-1}$ ) & 202.9(4.1) & $201.2(3.7)$ & 201.3(4.1) & 0.4 & 0.64 \\
\hline RER & $1.22(0.05)$ & $1.18(0.03)$ & $1.20(0.04)$ & 1.21 & 0.29 \\
\hline$E(W)$ & $780(38)$ & $860(22)^{a}$ & $880(19)^{a}$ & 4.1 & $<0.01$ \\
\hline Epinephrines $\left(\mathrm{nmol} \cdot \mathrm{L}^{-1}\right)$ & $2.78(0.3)$ & $2.01(0.3)^{a}$ & $1.78(0.3)^{a, b}$ & 8.6 & $<0.01$ \\
\hline Norepinephrines $\left(\mathrm{nmol} \cdot \mathrm{L}^{-1}\right)$ & $13.4(0.2)$ & $12.1(0.2)^{\mathrm{a}}$ & $11.3(0.2)^{a, b}$ & 19.4 & $<0.001$ \\
\hline
\end{tabular}

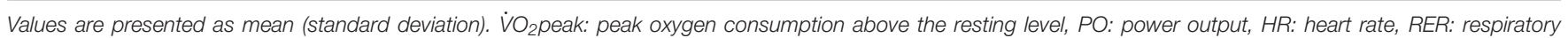
exchange ratio, E: energy consumption. ${ }^{a}$ Significant difference with NW ( $\left.p<0.01\right)$. ${ }^{b}$ Significant difference with OW (p < 0.01).

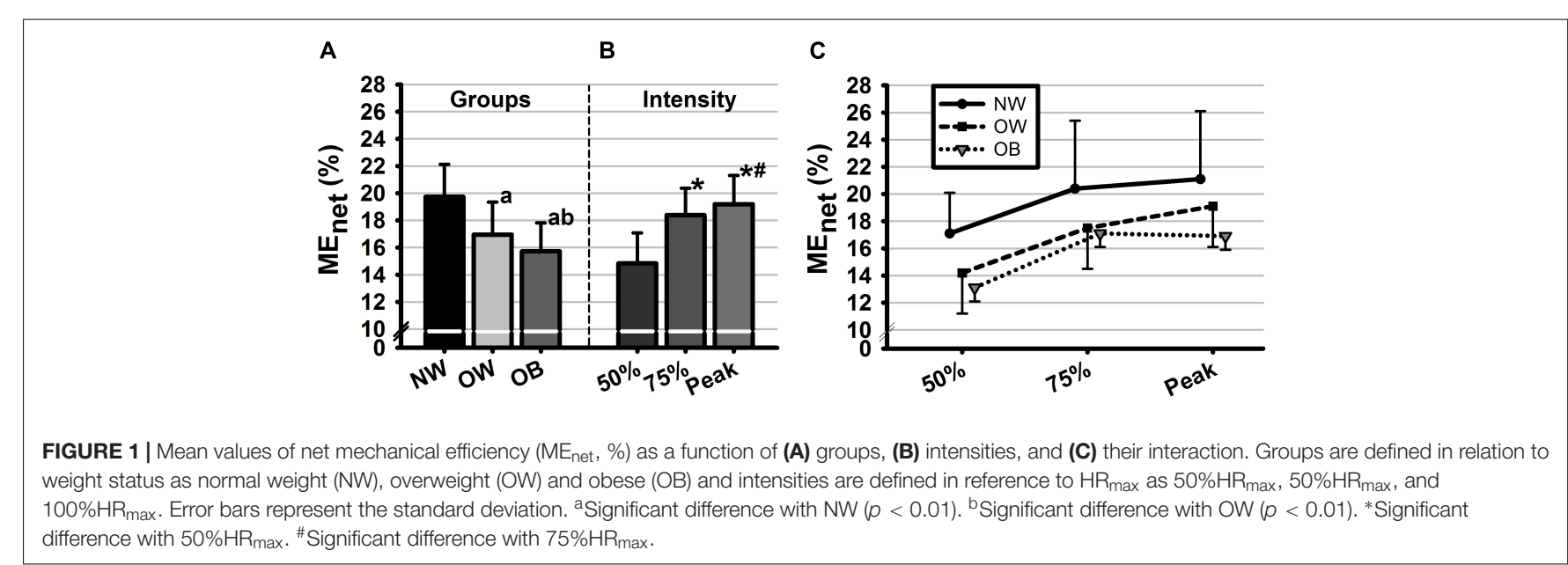

significant predictors of $\mathrm{ME}_{\text {net }}$ were also epinephrine $(ß=0.49$, $p=0.01)$, norepinephrine $(\beta=0.60, p<0.001)$ and power output $(ß=0.71, p<0.001)$.

\section{DISCUSSION}

To the best of our knowledge, this study was the first to investigate the relationship between $\mathrm{ME}$ and many potential underlying factors among obese $(\mathrm{OB})$, overweight (OW) and normal-weight (NW) adolescent boys when cycling at different exercise intensities of an incremental cycle test. Our results confirmed that (1) excess body fat had a significant effect in decreasing $\mathrm{ME}$ at all studied intensity levels. Moreover, (2) exercising at similar relative intensities brought about higher metabolic and physiological responses for the OB group that also presented the lowest ME values in comparison to the NW and OW groups. Finally, results showed that (3) body weight, \%LO and energy consumption were significant predictors of $\mathrm{ME}_{\text {net }}$ at the moderate intensity (i.e., 50\%HRmax), while this was no longer apparent at higher intensities where catecholamine levels and power output seemed to be better predictors of efficiency. The later finding was made possible by the choice of the studied population that presented different epinephrine and norepinephrine responses to an incremental exercise, a characteristic that was not addressed in previous studies examining factors of ME.

At rest and during all tested cycling intensity levels, absolute oxygen uptake (in $\mathrm{L} \cdot \mathrm{min}^{-1}$ ) was significantly higher for the OW and $\mathrm{OB}$ adolescents as compared to the NW adolescents (Nikolaidis et al., 2018), while no differences in HR values were detected between groups. This result might suggest a higher muscle oxygen extraction capacity per heart beat and/or a larger stroke volume for our obese adolescents (Salvadori et al., 1999; Lafortuna et al., 2006). The latter goes in line with previous reports on obese adult women (Lafortuna et al., 2006) and young obese adults (Salvadori et al., 1999), and could be interpreted in relation to the excess body mass and FFM. Furthermore, it has been suggested that increases in $\dot{\mathrm{VO}} \mathrm{O}_{2}$ and E during cycling in obese individuals can result from the extra work required to move the lower limbs (Anton-Kuchly et al., 1984) and the higher postural activity (Dempsey et al., 1966).

As hypothesized, obesity seems to affect metabolic and physiological responses to exercise in adolescents. Specifically, at $50 \% \mathrm{HRmax}$, our results showed a lower $\mathrm{ME}$ for the OB group in comparison to the OW and NW groups. As previously established by Butte et al. (2007), ME is negatively affected by 
energy expenditure rates at this intensity level in overweight children. These authors found that the higher energy expenditure in overweight children and adolescents were attributed in a large part to differences in body size and composition. Indeed, excess body weight, as presented in our $\mathrm{OB}$ adolescents, constitutes a major contributor for energy expenditure increases as more energy is consumed at a given work output (e.g., moderate aerobic level). Additionally, it was found that lipid oxidation rate (\%LO) was a significant predictor of $\mathrm{ME}$ at $50 \% \mathrm{HRmax}$, in a way that those having a high \%LO during the moderate aerobic stage were also more efficient. In fact, our $\mathrm{OB}$ and $\mathrm{OW}$ groups presented significantly higher respiratory exchange ratio values, which is a potential indicator of an impaired fat oxidation capability during exercise (van Baak, 1999). Accordingly, a recent study of Laaksonen et al. (2018) conducted in groups of subjects with different ME levels during cycling, showed a higher use of fatty acids for more efficient individuals during prolonged exercise at moderate exercise intensity. For these authors, the shift in relative contributions of fats and carbohydrates may explain the changes in ME. As assumed by Laaksonen et al. (2018), $\mathrm{ME}$ depends on the effectiveness of lipid oxidative capacity at moderate aerobic intensities. Despite that other complementary analyses are needed to confirm our assumption, the present study was the first to establish the link between \%LO and ME at moderate aerobic intensity among obese adolescents.

At higher intensity levels (i.e., 75\%HRmax and at $\dot{V} \mathrm{O}_{2 \text { peak }}$ ), subjects' efficiency increased as compared to its value at the moderate aerobic level (Figure 1B). This could be explained by the increases of both workload and the amount of energy consumed (Jabbour et al., 2013). At these two intensity levels (i.e., 75\%HRmax and $\mathrm{VO}_{2 \text { peak }}$ ), $\mathrm{ME}$ was negatively affected by body fat and weight status with the lowest values found for $\mathrm{OB}$ as compared to OW and NW adolescents. The lower ME observed for $\mathrm{OB}$ and $\mathrm{OW}$ groups may be a consequence of lower muscle performance. Accordingly, the power output developed at this stage was higher for NW as compared to $\mathrm{OB}$ and $\mathrm{OW}$ groups leading to increases in the magnitude of the numerator in the ME model, thus in the ME value. Furthermore, results revealed significantly lower epinephrine and norepinephrine responses to exercise for the $\mathrm{OB}$ and $\mathrm{OW}$ groups at all studied intensity levels. The latter supports previous findings on adolescents (Eliakim et al., 2006) showing a substantially attenuated catecholamine responses to bout of cycling exercise above the anaerobic threshold. Interestingly, the power output developed during 75\%HRmax and $\dot{V} \mathrm{O}_{2 \text { peak }}$ stages were significantly associated with epinephrine and norepinephrine concentrations, which was not the case at lower intensity. Indeed, as intensity increases, the reliance on fiber type-II to meet the imposed performance demand becomes greater (Sale, 1987), therefore the catecholamine responses are further stimulated by changes in acid-base balance and reduced oxygen availability to the working muscle (Schneider et al., 1992). The latter influences exercise performances by regulating muscular glycogenolysis (Richter et al., 1981).

Taken together, our results may offer a new insight in terms of ME's assessment, especially when exploring a high intensity exercise. Actually, the inclusion of the anaerobic energy production in ME's calculation is still unavailable and therefore not represented. Indeed, anaerobic contribution is increasingly involved in energy supply at intensities above the lactate threshold 2 (intensity corresponding to 75\%HRmax in our study). This might have limited our results, especially when comparing the three groups for which the anaerobic energy contribution at $75 \% \mathrm{HRmax}$ seemed to be the highest for the OB group (estimation simply based on lactate concentrations) potentially leading to an underestimation of ME. Moreover, ME was determined from incremental 2-min stages, which did not take into account potential differences in gas exchange kinetics between the three groups. Therefore, further studies are needed to include the anaerobic component in the determination of ME that would be examined at different steady state intensities to determine the extent of the phenomenon. Moreover, the use of net $\mathrm{ME}$ in this study, as opposed to gross $\mathrm{ME}$, allowed us to control for group differences in baseline (i.e., resting) energy expenditure, thus increasing the reliability of ME values. However, our method of ME calculation, similar to that considering the baseline as the energy cost of unloaded cycling (i.e., work efficiency), does not take into account variations in energy expenditure required to maintain homeostasis (Moseley and Jeukendrup, 2001). This also offers perspectives into calculation methods of cycling $\mathrm{ME}$ in relation to differences arising from group characteristics.

\section{CONCLUSION}

In conclusion, the present study highlights an important issue regarding predictors of $\mathrm{ME}$ in adolescent boys with different body fat percentages. It appears that underlying factors of ME may diverge according to the intensity of exercise. Our assumption of different underlying factors for ME is supported, and goes beyond the simple relation to the mass of body segments and the energy cost involved in movements (Lafortuna et al., 2006, 2009; Butte et al., 2007). Indeed, at moderate aerobic intensity, the energy consumption and lipid oxidative rates may be important factors contributing to lowering ME among obese and overweight individuals. At the contrary, at higher intensities, ME may be better explained by factors such as muscle power and catecholamine responses that are attenuated in obesity. Based on this relationship, further investigations are needed to provide a more complete profile regarding energy/metabolic forms (aerobic, anaerobic) to ensure that they are well represented in the ME model. From a practical standpoint and given the importance of $\mathrm{ME}$ as an indicator of exercise tolerance, it seems important to include both moderate- and high-intensity exercises to programs targeting obese adolescents, where different benefits would be expected.

\section{AUTHOR CONTRIBUTIONS}

GJ conceived and designed the study, collected the data, and drafted the manuscript. GJ and LM performed the data analysis and interpreted the data, and revised, read and approved the submitted version. 


\section{FUNDING}

The publication of this article was funded by the Qatar National Library.

\section{REFERENCES}

Anton-Kuchly, B., Roger, P., and Varene, P. (1984). Determinants of increased energy cost of submaximal exercise in obese subjects. J. Appl. Physiol. 56, 18-23. doi: 10.1152/jappl.1984.56.1.18

Boone, J., Koppo, K., Barstow, T. J., and Bouckaert, J. (2010). Aerobic fitness, muscle efficiency, and motor unit recruitment during ramp exercise. Med. Sci. Sports Exerc. 42, 402-408. doi: 10.1249/MSS.0b013e3181b of $2 \mathrm{e} 2$

Butte, N. F., Puyau, M. R., Vohra, F. A., Adolph, A. L., Mehta, N. R., and Zakeri, I. (2007). Body size, body composition, and metabolic profile explain higher energy expenditure in overweight children. J. Nutr. 137, 2660-2667. doi: $10.1093 /$ jn $/ 137.12 .2660$

Coyle, E. F., Sidossis, L. S., Horowitz, J. F., and Beltz, J. D. (1992). Cycling efficiency is related to the percentage of type I muscle fibers. Med. Sci. Sports Exerc. 24, 782-788. doi: 10.1249/00005768-199207000-00008

Craig, C. L., Marshall, A. L., Sjorstrom, M., Bauman, A. E., Booth, M. L., Ainsworth, B. E., et al. (2003). International physical activity questionnaire: 12-country reliability and validity. Med. Sci. Sports Exerc. 35, 1381-1395. doi: 10.1249/01. MSS.0000078924.61453.FB

Dempsey, J. A., Reddan, W., Balke, B., and Rankin, J. (1966). Work capacity determinants and physiologic cost of weight-supported work in obesity. J. Appl. Physiol. 21, 1815-1820. doi: 10.1152/jappl.1966.21.6.1815

Eliakim, A., Nemet, D., Zaldivar, F., McMurray, R. G., Culler, F. L., Galassetti, P., et al. (2006). Reduced exercise-associated response of the GH-IGF-I axis and catecholamines in obese children and adolescents. J. Appl. Physiol. 100, 16301637. doi: 10.1152/japplphysiol.01072.2005

Garby, L., and Astrup, A. (1987). The relationship between the respiratory quotient and the energy equivalent of oxygen during simultaneous glucose and lipid oxidation and lipogenesis. Acta Physiol. Scand. 129, 443-444. doi: 10.1111/j. 1365-201X.1987.tb10613.x

Jabbour, G., and Iancu, H. D. (2015). Mechanical efficiency improvement in relation to metabolic changes in sedentary obese adults. BMJ Open Sport Exerc. Med. 1:e000044. doi: 10.1136/bmjsem-2015-000044

Jabbour, G., Iancu, H. D., Mauriège, P., Joanisse, D. R., and Martin, L. J. (2017). High-intensity interval training improves performance in young and older individuals by increasing mechanical efficiency. Physiol. Rep. 5:e13232. doi: 10.14814/phy2.13232

Jabbour, G., Lambert, M., O’Loughlin, J., Tremblay, A., and Mathieu, M. E. (2013). Mechanical efficiency during a cycling test is not lower in children with excess body weight and low aerobic fitness. Obesity 21, 107-114. doi: 10.1002/oby. 20241

Jabbour, G., Lemoine-Morel, S., Casazza, G. A., Hala, Y., Moussa, E., and Zouhal, H. (2011). Catecholamine response to exercise in obese, overweight, and lean adolescent boys. Med. Sci. Sports Exerc. 43, 408-415. doi: 10.1249/MSS. 0b013e3181f1bef3

Koubi, H. E., Desplanches, D., Gabrielle, C., Cottet-Emard, J. M., Sempore, B., and Favier, R. J. (1991). Exercise endurance and fuel utilization: a reevaluation of the effects of fasting. J. Appl. Physiol. 70, 1337-1343. doi: 10.1152/jappl.1991.70.3. 1337

Kriketos, A. D., Baur, L. A., O’Connor, J., Carey, D., King, S., Caterson, I. D., et al. (1997). Muscle fibre type composition in infant and adult populations and relationships with obesity. Int. J. Obes. Relat. Metab. Disord. 21, 796-801. doi: 10.1038/sj.ijo.0800476

Laaksonen, M. S., Kyröläinen, H., Kemppainen, J., Knuuti, J., and Kalliokoski, K. K. (2018). Muscle free fatty-acid uptake associates to mechanical efficiency during exercise in humans. Front. Physiol. 9:1171. doi: 10.3389/fphys.2018. 01171

Lafortuna, C. L., Agosti, F., Busti, C., Galli, R., and Sartorio, A. (2009). The energy cost of cycling and aerobic performance of obese adolescent girls. J. Endocrinol. Invest. 32, 647-652. doi: 10.1007/BF03345735

\section{ACKNOWLEDGMENTS}

We are grateful for the cooperation and participation of the subjects.

Lafortuna, C. L., Proietti, M., Agosti, F., and Sartorio, A. (2006). The energy cost of cycling in young obese women. Eur. J. Appl. Physiol. 97, 16-25. doi: 10.1007/ s00421-006-0137-5

Layec, G., Haseler, L. J., Hoff, J., and Richardson, R. S. (2011). Evidence that a higher ATP cost of muscular contraction contributes to the lower mechanical efficiency associated with COPD: preliminary findings. Am. J. Physiol. Regul. Integr. Comp. Physiol. 300, R1142-R1147. doi: 10.1152/ajpregu.00835. 2010

McCarthy, H. D., Cole, T. J., Fry, T., Jebb, S. A., and Prentice, A. M. (2006). Body fat reference curves for children. Int. J. Obes. 30, 598-602. doi: 10.1038/sj.ijo. 0803232

McGilvery, R. W., and Goldstein, G. W. (1983). Biochemistry: A Function Approach. Philadelphia, PA: Saunders, 810-976.

Moseley, L., and Jeukendrup, A. E. (2001). The reliability of cycling efficiency. Med. Sci. Sports Exerc. 33, 621-627. doi: 10.14814/phy2. 13233

Nikolaidis, P. T., Kintziou, E., Georgoudis, G., Afonso, J., Vancini, R., and Knechtle, B. (2018). The Effect of body mass index on acute cardiometabolic responses to graded exercise testing in children: a narrative review. Sports 6:103. doi: 10.3390/sports6040103

Richter, E. A., Sonne, B., Christensen, N. J., and Galbo, H. (1981). Role of epinephrine for muscular glycogenolysis and pancreatic hormonal secretion in running rats. Am. J. Physiol. 240, E526-E532. doi: 10.1152/ajpendo.1981.240.5. E526

Sale, D. G. (1987). Influence of exercise and training on motor unit activation. Exerc. Sport Sci. Rev. 15, 95-151. doi: 10.1249/00003677-19870015000008

Salvadori, A., Fanari, P., Fontana, M., Buontempi, L., Saezza, A., Baudo, S., et al. (1999). Oxygen uptake and cardiac performance in obese and normal subjects during exercise. Respiration 66, 25-33. doi: 10.1159/00002 9333

Schneider, D. A., McGuiggin, M. E., and Kamimori, G. H. (1992). A comparison of the blood lactate and plasma catecholamine thresholds in untrained male subjects. Int. J. Sports Med. 13, 562-566. doi: 10.1055/s-2007-102 4565

Slaughter, M. H., Lohman, T. G., Boileau, R., Horswill, C. A., Stillman, R. J., Van Loan, M. D., et al. (1988). Skinfold equations for estimation of body fatness in children and youth. Hum. Biol. 60, 709-723.

Spiro, S. G. (1979). Exercise testing in clinical medicine. Br. J. Dis. Chest 71, 145-172. doi: 10.1016/0007-0971(77)90106-1

Strobel, G., Friedmann, B., Siebold, R., and Bärtsch, P. (1999). Effect of severe exercise on plasma catecholamines in differently trained athletes. Med. Sci. Sports Exerc. 31, 560-565. doi: 10.1097/00005768-19990400000011

Tanner, J. (1962). Growth at Adolescence. Oxford: Blackwell Scientific Publications. van Baak, M. A. (1999). Exercise training and substrate utilisation in obesity. Int. J. Obes. Relat. Metab. Disord. 23, S11-S17. doi: 10.1038/sj.ijo.08 00879

Van Beaumont, W. (1972). Evaluation of hemoconcentration from hematocrit measurements. J. Appl. Physiol. 32, 712-713. doi: 10.1152/jappl.1972.32.5.712

Vettor, R., Macor, C., Rossi, E., Piemonte, G., and Federspil, G. (1997). Impaired counterregulatory hormonal and metabolic response to exhaustive exercise in obese subjects. Acta Diabetol. 34, 61-66. doi: 10.1007/s00592005 0068

Villelabeitia-Jaureguizar, K., Vicente-Campos, D., Berenguel Senen, A., Verónica Hernández, Jiménez, V., Lorena Ruiz, et al. (2018). Mechanical efficiency of high versus moderate intensity aerobic exercise in coronary heart disease patients: a randomized clinical trial. Cardiol. J. doi: 10.5603/CJ.a2018.0052 [Epub ahead of print].

Weinstein, Y., Kamerman, T., Berry, E., and Falk, B. (2004). Mechanical efficiency of normal-weight prepubertal boys predisposed to obesity. 
Med. Sci. Sports Exerc. 36, 567-573. doi: 10.1249/01.MSS.0000121958.99 985.A5

Conflict of Interest Statement: The authors declare that the research was conducted in the absence of any commercial or financial relationships that could be construed as a potential conflict of interest.
Copyright (c) 2019 Jabbour and Majed. This is an open-access article distributed under the terms of the Creative Commons Attribution License (CC BY). The use, distribution or reproduction in other forums is permitted, provided the original author(s) and the copyright owner(s) are credited and that the original publication in this journal is cited, in accordance with accepted academic practice. No use, distribution or reproduction is permitted which does not comply with these terms. 